\title{
Plädoyer für ein arbeits- und sozialpolitisches Mandat - Ergebnisse einer Beschäftigten- befragung der IG Metall
}

\author{
HANS-JÜRGEN URBAN, CHRISTOPH EHLSCHEID
}

Schon im Wahlkampf und auch im Nachgang zur Bundestagswahl 2013 berichteten die Medien ausführlich über Personen, Koalitionen und Ministerposten, wenig über soziale Problemlagen und politische Antworten. Augenfällig war eine eklatante Diskrepanz zwischen dem Problemdruck und den Erwartungen der Bevölkerung gegenüber den politischen Entscheidern einerseits und den Schwerpunkten der öffentlichen Diskurse andererseits. Die Kluft zwischen den Erwartungen der Menschen und den politischen Diskursen zeigt sich deutlich in den zentralen Ergebnissen einer aktuellen Beschäftigtenbefragung.

\section{Die Beschäftigtenbefragung der IG Metall}

Zwischen Mai und August 2013 führte die IG Metall eine Beschäftigtenbefragung durch. ${ }^{1}$ Mehr als eine halbe Million der Befragten antworteten ( $\mathrm{n}=514$ 134), darunter etwa ein Drittel unorganisierte Beschäftigte (31\%). Der vierseitige Fragebogen umfasste 20 Fragenkomplexe mit über 70 Einzelfragen zu verschiedenen arbeits- und gesellschaftspolitischen Themen. Da die Befragung als offenes und beteiligungsorientiertes Projekt in den Betrieben durchgeführt wurde, können die Ergebnisse nach der Methodenlehre der quantitativen Sozialforschung zwar nicht als repräsentativ gelten. Gleichwohl entspricht die Verteilung der Antworten nach Alter, Geschlecht, Qualifikation und regionaler Konzentration in etwa der Beschäftigten- und Branchenstruktur des Organisationsbereichs der IG Metall. In dieses Bild passt auch, dass über $50 \%$ des Rücklaufs aus den mitgliederstarken und aktiven IG Metall-Bezirken Bayern und BadenWürttemberg stammen. ${ }^{2}$

Insgesamt vermitteln die Antworten ein aussagefähiges Bild über die Einschätzungen der Beschäftigten zu ihren Arbeitsbedingungen, ihren Arbeitsansprüchen und ihren Erwartungen an die Prioritäten der gewerkschaftlichen Politik. Sie liefern damit wichtige Hinweise für die Weiterentwicklung gewerkschaftlicher Programmatik und Praxis im Allgemeinen, für die Definition von Handlungsfeldern und Forderungen im Besonderen. Dabei stellen Mitgliederund Beschäftigungsbefragungen mittlerweile ein Standar- dinstrument im Rahmen der strategischen Planung der IG Metall dar. Genutzt werden dabei vor allem die Möglichkeiten der Online-Kommunikation. Sie dienen der VorabErfassung von Stimmungen und Meinungsbildern in den Belegschaften sowie der schnellen Rückkoppelung organisationsinterner Debattenstände mit den Präferenzen der Mitgliederbasis oder der Belegschaften und erweisen sich zunehmend als ein effektives Mittel einer mitgliedernahen Meinungsbildung und Entscheidungsfindung. Im Folgenden sollen einige Befragungsergebnisse in den Feldern der Arbeits- und Sozialpolitik vorgestellt und erste Schlussfolgerungen gezogen werden.

\section{Politikfeld Arbeitsmarkt}

Trotz guter Konjunktur und vergleichsweise niedriger Arbeitslosenzahlen machen sich fast $40 \%$ der Befragten große oder sogar sehr große Sorgen um ihren Arbeitsplatz; und das, obwohl fast $90 \%$ der Befragten angeben, einen unbefristeten Arbeitsvertrag zu haben. In diesen Befürchtungen spiegelt sich möglicherweise eine wachsende Unsicherheit auch bei Stammbeschäftigten wider, die ihre Ursachen in mehreren Faktoren haben kann: So haben viele Belegschaften in den letzten Jahren die Erfahrung machen müssen, dass in einer globalisierten Ökonomie mit ihren erweiterten Verlagerungsmöglichkeiten und den kurzfristigen Renditeerwartungen der Finanzmärkte jeder Arbeitsplatz potenziell als bedroht gelten muss. Und das nicht nur in Krisen-

1 Die wichtigsten Ergebnisse der Befragung finden sich in IG Metall Vorstand (Hrsg.) (2013): Arbeit: sicher und fair! Die Befragung. Ergebnisse, Zahlen, Fakten, Frankfurt a. M.

$241 \%$ der Antworten kommen aus dem Bereich der Produktion (Fertigung/Montage), $21 \%$ aus den produktionsnahen Bereichen (Logistik, Arbeitsvorbereitung, Qualitätssicherung, Instandhaltung), $12 \%$ aus Forschung und Entwicklung, $8 \%$ aus Einkauf, Vertrieb, Marketing, $7 \%$ interne Dienstleistungen (IT, Personal, Controlling) und $11 \%$ sind Sonstige. 
unternehmen und Krisenbranchen. Die Ängste, die diese „Krise in Permanenz“ auslöst, werden zusätzlich noch durch den Umstand befeuert, dass mit der voranschreitenden Ausbreitung prekärer Arbeit und dem Abbau sozialstaatlicher Leistungen für Arbeitslose die Furcht wächst, mit dem eigenen Arbeitsplatz die soziale Absicherung zu verlieren.

Angesichts der Zunahme von Leiharbeit, Befristungen und Werkverträgen und der Ausweitung des Niedriglohnsektors kann es nicht verwundern, wenn sich die Befragten eindeutig für eine Neuordnung des Arbeitsmarktes aussprechen. So halten mehr als $90 \%$ die „Eingrenzung des Niedriglohnsektors, der Leiharbeit und von Mini-Jobs u.a. “ für wichtig bis sehr wichtig. Die Forderungen der Befragten bestätigen die IG Metall, mit ihrer Kampagne „Arbeit: sicher und fair“ Themen aufgegriffen zu haben, die aus Sicht der Beschäftigten von hoher Relevanz sind. Notwendig ist eine solidarische Neuordnung des Arbeitsmarktes, die darauf zielt, Prekarität und Armut für Beschäftigte und Arbeitslose zu vermeiden und für beide Perspektiven und Sicherheit zu schaffen. Unverzichtbar ist daher eine grundlegende Reform der Arbeits- und Sozialverfassung. Elemente einer entsprechenden Reformstrategie sind die Einführung eines gesetzlichen, flächendeckenden Mindestlohns sowie die Regulierung von Leiharbeit und Werkverträgen und die Durchsetzung des Grundsatzes „Gleiche Arbeit, gleiches Geld, gleiche Rechte“. Ebenso notwendig ist aber auch eine Abkehr vom aktivierenden Arbeitsmarktsystem mit seinen nicht existenzsichernden Lohnersatzleistungen und seinen repressiven Zumutbarkeitsregeln.

Die Gewerkschaften tun gut daran, diese Forderungen weiterhin weit oben auf ihrer politischen Agenda zu platzieren und gegenüber der neuen Bundesregierung mit Nachdruck zu vertreten. Denn die Auseinandersetzung um die Zukunft der Arbeits- und Sozialverfassung hat für die Gewerkschaften auch eine machtpolitische Dimension: So hat die Deregulierung des Arbeitsmarktes zu einer massiven Schwächung der Verhandlungs- und Organisationsmacht von betrieblichen Interessenvertretungen und Gewerkschaften geführt. Die gezielte Förderung prekärer Beschäftigung und verschärfte Regelungen beim Sozialleistungsbezug haben den Konkurrenzdruck zwischen Prekariat und Stammbelegschaften sowie zwischen Beschäftigten und Arbeitslosen deutlich erhöht. ${ }^{3}$

\section{Politikfeld Arbeitspolitik}

Nahezu $80 \%$ der Befragten berichten über zunehmende Arbeitsverdichtung. Knapp über die Hälfte der Befragten, nämlich $51 \%$, fühlen sich ständig oder häufig gehetzt oder unter Zeitdruck. Ständig oder häufig arbeiten $21 \%$ außerhalb der regulären Arbeitszeit. 12 \% geben an, häufig oder ständig in der Freizeit für den Betrieb erreichbar bleiben zu müssen. Dem steht der Wunsch der Beschäftigten gegenüber, die zu $77 \%$ gänzlich oder eher „eine geregelte Arbeitszeit mit klar festgelegtem Beginn und Ende" einfordern, um Arbeit und Privatleben besser vereinbaren zu können.
Alle Anzeichen sprechen dafür, Fragen der Leistungsbedingungen und der Gesundheit zu einem zentralen Bestandteil der gewerkschaftlichen Strategie zu machen. Eine weitere Zuspitzung der Belastungen und Zumutungen droht, den seidenen Faden der Gesundheit zum Reißen zu bringen, der in vielen Befunden schon als angespannt geschildert wird. Das kann aber auch bedeuten: Der Kampf um Gesundheit in der Arbeit könnte zu einem zentralen Konfliktfeld werden, in dem erhebliche Mobilisierungspotenziale für die Gewerkschaften verborgen liegen.

Diese werden sich nur erschließen lassen, wenn es gelingt, Präventionsbewegung von unten $\mathrm{zu}$ initiieren. Gefordert sind dabei Betriebsräte und gewerkschaftliche Vertrauensleute. Gemeinsam gilt es, Bewusstsein und Handlungsbereitschaft gegen Gefährdungen bei der Arbeit zu fördern, Beschäftigte als Experten ihrer Arbeitsbedingungen einzubeziehen und Projekte „Guter Arbeit“ betrieblich zu verankern.

Hinzukommen muss aber auch eine Verbesserung der rechtlichen Rahmenbedingungen. So fehlen im Bereich der psychischen Belastungen eindeutige und verbindliche Vorgaben im Arbeitsschutzrecht. Deshalb hat die IG Metall die Initiative ergriffen und den viel beachteten Entwurf einer konkretisierenden Arbeitsschutzverordnung („Anti-StressVerordnung“) vorgelegt. Der IG Metall ist bei ihrer Initiative zum Thema psychische Belastungen in der modernen Arbeitswelt ein erfolgreiches Agenda-Setting gelungen. In der kommenden Legislaturperiode wird es nun darauf ankommen, den Druck auf die Politik zur Verabschiedung einer entsprechenden Verordnung hochzuhalten. Die Chancen für einen gewerkschaftlichen Erfolg stehen dabei nicht schlecht. $^{4}$

\section{Politikfeld Demografie}

In der wissenschaftlichen wie politischen Debatte besteht ein weitgehender Konsens, dass die absehbare „Alterung“ von Belegschaften und Gesellschaft den arbeitspolitischen Problemdruck in den Betrieben erhöhen wird. Die Befunde der Beschäftigtenbefragung weisen darauf hin, dass die Betriebe nicht in ausreichendem Maße auf die Herausforderungen des demografischen Wandels vorbereitet

3 In einschlägigen Studien wird der (zweifelhafte) Erfolg der Maßnahmen konstatiert. So stellt das Institut für Arbeitsmarkt- und Berufsforschung fest, dass sich Arbeitsuchende infolge des Hartz-IV-Regimes häufiger als früher auch um inadäquate Arbeitsplätze bemühen. Vgl. Kettner, A./Rebien, M. (2007): Hartz IV-Reform. Impulse für den Arbeitsmarkt, in: IAB Kurzbericht 19/2007.

4 Zum Stand der Umsetzung der Anti-Stress-Initiative der IG Metall vgl. Urban, H.-J./Fergen, A. (2014): Politische Zwischenbilanz der Anti-Stress-Initiativen, in: Schröder, L./Urban, H.-J. (Hrsg.): Gute Arbeit. Jahrbuch 2014. Profile prekärer Arbeit - Arbeitspolitik von unten, Frankfurt a. M. (im Erscheinen). 
sind. Altersgerechte Arbeitsplätze sind, trotz anderslautender Bekundungen von Arbeitgebern und Politikern, Mangelware. So glauben gerade mal $4 \%$ der Befragten, dass ihr Betrieb sehr gut auf älter werdende Belegschaften vorbereitet sei. Und 48 \% der Beschäftigten sehen ihren Betrieb schlecht oder gar nicht vorbereitet. Unter solchen Bedingungen verwundert es auch nicht, dass gerade mal $31 \%$ der Beschäftigten davon ausgehen, bis zum gesetzlichen Renteneintrittsalter arbeiten zu können. Bei Beschäftigten, die besonders belastenden Arbeitsbedingungen ausgesetzt sind, fallen die Ergebnisse noch schlechter aus. Das gilt etwa für Schichtarbeiter.

Diese Befunde unterstreichen die Bedeutung des demografischen Wandels als wichtiges gewerkschaftliches $\mathrm{Zu}$ kunftsthema. Die IG Metall hat angesichts des wachsenden Problemdrucks und der hohen Bedeutung des Themas für ihre Mitglieder die Initiative ergriffen und im Rahmen ihrer Kampagne „Gute Arbeit - gut in Rente“ die Missstände in der Arbeitswelt und den Mangel an Arbeitsplätzen für Ältere zum Gegenstand betrieblichen Handelns gemacht. In den kommenden Jahren wird es darauf ankommen, den aus der Beschäftigtenbefragung deutlich gewordenen Handlungsdruck aufzugreifen und erste erfolgreiche Ansätze in einzelnen Betrieben in eine breit angelegte Initiative für eine „demografiesensible“ Arbeitswelt einmünden zu lassen.

\section{Politikfeld Alterssicherung}

Die Beschäftigten haben große Sorgen um ihre Absicherung im Alter. Gerade einmal $4 \%$ der Befragten glauben, dass sie einmal von ihrer gesetzlichen Rente „gut“ werden leben können. Ein gutes Drittel geht von einer Rente aus, die "gerade ausreichen" wird. Mehrheitlich dominiert die Sorge, dass die Altersbezüge aus der gesetzlichen Rentenkasse nicht reichen werden. Das sagen $42 \%$ der Befragten, und bei den unter 35-Jährigen haben sogar 52 \% keine Hoffnung auf eine auskömmliche Rente. Die geäußerten Befürchtungen sind nicht unbegründet: Der Um- und Abbau der Rentenversicherung hat im Zuge einer ganzen Serie von sogenannten Reformen für schwerwiegende Leistungskürzungen gesorgt, eine weitreichende (Teil)Privatisierung der Alterssicherung vorangetrieben und stufenweise die Regelaltersgrenzen angehoben. Die langfristigen Folgen sind absehbar: Lebensstandardsicherung im Alter rückt für viele in weite Ferne und bis in den Kern der arbeitnehmerischen Mitte hinein droht Altersarmut wieder zu einem ernsthaften Risiko zu werden. ${ }^{5}$ Dass die private Vorsorge die Lücken in der gesetzlichen Rentenversicherung nicht schließen kann, ist offensichtlich. Auch in den Einschätzungen der Betroffenen findet das seinen Niederschlag. So glauben denn auch lediglich $28 \%$, dass sie für ihr Alter in ,ausreichendem Umfang“ zusätzlich vorsorgen. Der Anteil derer, die gar keine zusätzliche Altersvorsorge betreiben, weil sie es sich nicht leisten können, ist bei Beschäftigten ohne Berufsausbildung mit $40 \%$ besonders hoch. An der Problemlage der unteren Einkommensgruppe zeigen sich die Grenzen der privaten Vorsorge besonders deutlich. Gerade diejenigen Gruppen, die angesichts des sinkenden Rentenniveaus am stärksten auf die zusätzliche Vorsorge angewiesen wären, können sie sich schlicht nicht leisten.

Aus Sorge um ihre Sicherung im Alter wenden sich fast $90 \%$ gegen die Absenkung des Rentenniveaus und über $90 \%$ wollen, dass die „Rente mit 67 “ zurückgenommen wird. Zudem sprechen sich die Beschäftigten deutlich für flexible Ausstiegsmöglichkeiten am Ende des Erwerbslebens aus. Die Möglichkeit, früher aus dem Erwerbsleben auszusteigen, halten $92 \%$ der Befragten für wichtig oder sehr wichtig. Zudem fordern $98 \%$ einen abschlagsfreien Ausstieg nach „langer Erwerbstätigkeit“ sowie die Förderung eines flexiblen Ausstiegs durch den Gesetzgeber. Im Organisationsbereich der IG Metall ist die Altersteilzeit ein wichtiges Instrument, um einen vorzeitigen Ausstieg zu fairen Bedingungen zu ermöglichen. Auch das zeigen die Antworten. So würden $53 \%$ der Befragten gerne die heutige Altersteilzeitregelung nutzen. Dem hohen Interesse stehen aber auch $20 \%$ der Beschäftigten gegenüber, die sich die Altersteilzeit aus finanziellen Gründen nicht leisten können, und $16 \%$, die die Regelung gar nicht kennen. Das spricht für eine stark selektive Wirkung der Altersteilzeitregelung und verweist sowohl auf den tarifpolitischen Handlungsbedarf als auch auf die Bedeutung der öffentlichen Förderung der Altersteilzeit. So sind mit dem Wegfall der Förderung der Bundesagentur für Arbeit (Ende 2009) in den Handwerksbranchen viele Arbeitgeber aus der Altersteilzeit ausgestiegen und bieten betrieblich keine Regelung mehr an.

\section{Gewerkschaftspolitische Schlussfolgerungen}

Die Ergebnisse der Befragung liefern einen umfassenden Fundus an Einschätzungen, Erwartungen und Präferenzen in den Betrieben des Organisationsbereiches der IG Metall. Ihre gewerkschaftspolitische Auswertung hat erst begonnen. Mit Blick auf die hier skizzierten Ergebnisse lassen sich Schlussfolgerungen für das politische Selbstverständnis der IG Metall ziehen und Hinweise auf Defizite im Kernfeld gewerkschaftlicher Leistungspolitik finden. Zudem ist der Aufklärungs- und Diskussionsbedarf beim Thema Krisenpolitik in Europa nicht zu übersehen.

(1) Votum für ein sozialpolitisches Mandat der IG Metall: Die Einstellungen der Beschäftigten zu den Themen Rente und Altersübergang sind ebenso wie die Antworten zu den Bedingungen altersgerechten Arbeitens eine klare Bestätigung der Forderungen, für die sich die IG Metall in ihrer Kampagne „Gute Arbeit - Gut in Rente“ seit Monaten engagiert. Die Befragten weisen bei der Durchsetzung der

5 Zur Krise der Alterssicherung vgl. Urban, H.-J./Ehlscheid, Ch./Gerntke, A. (Hrsg.) (2010): Der Neue Generationenvertrag. Sozialstaatliche Erneuerung in der Krise, Hamburg. 
Forderungen gerade auch dem Feld der Weiterentwicklung der sozialen Sicherungssysteme eine wichtige Rolle zu. So halten 97 \% der Befragten den Erhalt und die Stärkung der sozialen Sicherungssysteme für ein wichtiges oder sehr wichtiges Handlungsfeld. Das ist ein starkes Votum für ein sozialpolitisches Mandat der Gewerkschaften. Die IG Metall kann bei der Fortsetzung ihrer Kampagne gegen die Rente mit 67 und für flexible Ausstiegsmöglichkeiten sicher sein, dass sie damit die Erwartungen der Mitglieder erfüllt und auf breite Unterstützung trifft. Mit ihren Vorschlägen für neue Rentenarten, die einen erleichterten Rentenzugang für Erwerbsgeminderte ermöglichen, eine öffentliche Förderung gleitender Übergänge (neue, öffentlich geförderte Altersteilzeit) sicherstellen und einen abschlagsfreien Rentenzugang für Beschäftigte mit langen Versicherungszeiten eröffnen, hat die IG Metall konkrete Vorstellungen für die rechtliche Umsetzung entwickelt. Sie bilden die Grundlage für die weitere politische Mobilisierung und den Dialog mit der Politik.

(2) Schutz vor Leistungsdruck und Arbeitsverdichtung - ein defizitäres Politikfeld: Die Befunde der Beschäftigtenbefragung zu den angespannten Arbeits- und Leistungsbedingungen bestätigen den Trend zur „Entgrenzung“ von Arbeitszeit und Leistung. Stress, Arbeitshetze und „Arbeiten ohne Ende" bestimmen den Arbeitsalltag von immer mehr Menschen. Offensichtlich hat sich ein neuer Modus der Leistungssteuerung durchgesetzt - mit einer neuen Maßlosigkeit, die Grenzen der menschlichen Leistungsfähigkeit negiert und menschliche Arbeitskraft internen Märkten unterordnet.

Auf diese Entwicklungen müssen die Gewerkschaften reagieren, wollen sie ein relevanter Akteur der Regulierung der Arbeitsbedingungen bleiben. Das gilt nicht zuletzt für die IG Metall, die sich in der Vergangenheit durch die Vereinbarung von Mantel- und Lohnrahmentarifverträgen und bei betrieblichen Leistungsvereinbarungen (Akkord und Prämienlohn) politisch profilieren konnte. Die Stabilisierung dieses Profils erfordert zweifelsohne eine erneute Aktivierung gewerkschaftlicher Leistungspolitik. Doch offensichtlich klafft zwischen der Diagnose und der Erarbeitung einer wirksamen gewerkschaftlichen Strategie (noch) eine Lücke. Die Konturen einer neuen Leistungspolitik, die dem umfassenden unternehmerischen Zugriff auf die Arbeitsleistung der Beschäftigten in allen Bereichen Grenzen setzt und einen zeitgemäßen Modus der Leistungsregulation etabliert, sind bislang nicht erkennbar. Insofern können die Befragungsergebnisse als Mahnung interpretiert werden, mit der Leistungspolitik ein traditionelles Kernfeld gewerkschaftlicher Betriebs- und Tarifpolitik neu zu entdecken und zu erschließen. Bei dieser Suchbewegung wird es nicht nur - vielleicht nicht mal in erster Linie - darum gehen, an neuen Methoden der Leistungsbewertung und -bemessung $\mathrm{zu}$ arbeiten. ${ }^{6}$ Vordringlich ist eine ehrliche Auseinandersetzung mit der Frage, wie gegen den immensen Wettbewerbsund Produktivitätsdruck in den Unternehmen zumutbare
Leistungsvorgaben und eine angemessene Personalbemessung durchgesetzt werden können.

(3) Aufklärungs- und Diskussionsbedarf beim Thema Krisenpolitik in Europa: Trotz der Wucht, mit der die Finanz-, Fiskal- und Wirtschaftskrise über die Eurozone hereingebrochen ist, und trotz der weitreichenden ökonomischen, sozialen und politischen Verwerfungen, die die neoliberale Austeritätspolitik ausgelöst hat, scheint die europäische Krise aus Sicht der Beschäftigten recht weit weg zu sein. So halten rund ein Fünftel der Befragten eine solidarische Krisenbewältigung in Europa für weniger wichtig oder unwichtig. Mit $40 \%$ halten weniger als die Hälfte der Beschäftigten Europa für ein sehr wichtiges Handlungsfeld für den von der IG Metall geforderten Kurswechsel (Tabelle 1). Damit wird der europäischen Frage zwar von einer ansehnlichen Zahl der Befragten eine große Bedeutung beigemessen, aber verglichen mit anderen abgefragten Themen rangiert die Bewältigung der europäischen Krise auf dem letzten Rang. Die Erklärung für diese relative Distanz der Beschäftigten zur europäischen Krise lässt sich angesichts der gewählten Fragestellungen nicht in der Beschäftigtenbefragung der IG Metall finden. Zu vermuten ist aber, dass sich die Befragten aufgrund der aktuellen wirtschaftlichen Situation in Deutschland und der Bedingungen am Arbeitsmarkt nicht als unmittelbar Krisenbetroffene sehen. Die Eurokrise ist aus dieser Sicht die Krise der verschuldeten Südländer und scheint damit weit weg von den Arbeits- und Lebensverhältnissen in Deutschland zu sein. Eine solche Deutung legt auch eine Umfrage von Infratest/dimap im Auftrag der ARD nahe. So stimmten $73 \%$ der Wahlberechtigten im Oktober der Aussage zu: „Ich bin persönlich von der Krise bisher nicht betroffen"?

Auch wenn sich die Befragten von der europäischen Krise wenig betroffen fühlen, darf der objektive Problemdruck, der von der aktuellen Krisenpolitik ausgeht, nicht unterschätzt werden. Derzeit wird in Europa eine institutionelle Struktur errichtet, die den neoliberalen Sparkurs zementiert und zunehmend Tarifsysteme und Sozialstandards unterminiert. Die Gewerkschaften stehen kurzfristig vor der Aufgabe, den Widerstand gegen eine ökonomisch und sozial desaströse Krisenpolitik europaweit zu organisieren. Mittelfristig bedarf es einer europapolitischen Neuorientierung der Gewerkschaften. Notwendig sind eine effizientere Koordinierung der nationalen Verteilungs- und Arbeitsmarktpolitiken und zugleich der Ausbau der Interessenvertretungsstrukturen in europäischen Konzer-

6 Zu Fragen einer zeitgemäßen Leistungspolitik vgl. Ehlscheid, Ch./Nobel, U. (2008): Von der Diagnose zurTherapie? Neue Leistungspolitik (auch) in indirekten Bereichen, in: Wagner, H. (Hrsg.): Arbeit und Leistung - gestern und heute: ein gewerkschaftliches Politikfeld, Hamburg.

7 www.wahl.tagesschau.de 
TABELLE 1

\section{Wichtige Handlungsfelder für einen politischen Kurswechsel}

Anteile in Prozent

Verteilung der Antworten auf die Frage: Die IG Metall fordert einen Kurswechsel in der Politik. Wie wichtig sind für Sie folgende Handlungsfelder?

\begin{tabular}{|c|c|c|c|c|}
\hline & sehr wichtig & wichtig & weniger wichtig & unwichtig \\
\hline Regulierung der Finanzmärkte und Besteuerung von Finanztransaktionen & 54 & 35 & 9 & 2 \\
\hline $\begin{array}{l}\text { Mehr Verteilungsgerechtigkeit durch eine stärkere Besteuerung hoher } \\
\text { Einkommen, hoher Vermögen und großer Erbschaften }\end{array}$ & 47 & 34 & 14 & 4 \\
\hline $\begin{array}{l}\text { Erhalt und Stärkung der sozialen Sicherungssysteme (Rente, Pflege, } \\
\text { Gesundheit, Arbeitslosenversicherung) }\end{array}$ & 75 & 22 & 2 & 0 \\
\hline Förderung der Gleichstellung von Frauen und Männern & 48 & 35 & 13 & 5 \\
\hline Gleiche Bildungschancen unabhängig von Herkunft und Einkommen der Eltern & 67 & 27 & 4 & 1 \\
\hline Ein ökologisch und sozial nachhaltiges Wachstum & 53 & 41 & 5 & 1 \\
\hline Eine solidarische Krisenbewältigung in Europa & 40 & 40 & 15 & 5 \\
\hline
\end{tabular}

Quelle: IG Metall Vorstand (Hrsg.) (2013): Arbeit: sicher und fair! Die Befragung. Ergebnisse, Zahlen, Fakten, Frankfurt a. M.

nen. Diese Aufgaben werden sich von den Gewerkschaften nur meistern lassen, wenn sie größere Anstrengungen unternehmen, den Dialog mit den Beschäftigten über die tatsächlichen Krisenursachen zu führen und die weitreichenden Folgen der neoliberalen Krisenpolitik aufzuklären.

\section{Ausblick}

Die bisherige Auswertung belegt den hohen Gebrauchswert der Befragungsergebnisse für eine mitgliedernahe Interessenpolitik. Einerseits bestätigen sie die IG Metall in ihrem Engagement für eine sozialstaatliche Neuordnung des Arbeitsmarktes, gegen prekäre Arbeit und für alternsgerechte Arbeitsbedingungen sowie gegen die Rente mit 67 und für flexible Übergänge in den Ruhestand. Andererseits weisen sie auf Schwachstellen und Defizite der bisherigen Prioritätensetzungen hin. Das gilt zweifelsohne mit Blick auf die Aufklärungs- und Diskussionsnotwendigkeiten bezüglich einer akzeptablen Krisenpolitik in Europa sowie auf die Begrenzung von Leistungsdruck und Arbeitshetze in den Betrieben. Hier werden Bedarfe in den Feldern der transnationalen Fiskal- und Wirtschaftspolitik sowie der qualitativen Tarifpolitik deutlich.

Insgesamt steht der Umgang der Gewerkschaften mit Ad-hoc-Meinungsbildern aus den Betrieben, wie sie durch Befragungen dieser Art erstellt werden können, noch am Anfang. Sie liefern einerseits schnelle und authentische Informationen an die gewerkschaftlichen Strategieabteilungen und können das Risiko politischer Schwerpunktsetzungen jenseits der Mitgliedererwartungen reduzieren. Zugleich können etwaige Diskrepanzen zwischen Mitgliedererwartungen und gewerkschaftlicher Praxis auf Defizite in der gewerkschaftsinternen Entscheidungsfindung verweisen, die nur über ein Mehr an Beteiligung und Diskussion zu beseitigen sein dürften. Fazit: Mitglieder- und Beschäftigtenbefragungen stellen ein innovatives Element in der Mitglieder-Organisations-Kommunikation dar, das - adäquat genutzt - die Legitimation gewerkschaftlicher Interessenvertretung erhöhen und eine offene Debattenkultur beleben kann.

\section{AUTOREN}

HANS-JÜRGEN URBAN, Dr. phil., ist geschäftsführendes Vorstandsmitglied der IG Metall. Arbeitsschwerpunkte: Arbeitsmarkt- und Sozialpolitik, Berufliche Bildung sowie Arbeits- und Gesundheitsschutz, Perspektiven des Europäischen Sozialmodells, Gewerkschaftsforschung und Strategiefragen gewerkschaftlicher Politik.

hans-juergen.urban@igmetall.de

CHRISTOPH EHLSCHEID, Politikwissenschaftler, ist Bereichsleiter Sozialpolitik beim Vorstand der IG Metall. Arbeitsschwerpunkte: Weiterentwicklung der Sozialsysteme, Perspektiven der Alterssicherung und Strategiefragen gewerkschaftlicher Politik.

christoph.ehlscheid@igmetall.de 